\title{
The influence of surfaces and interfaces on coherent phonons in semiconductors
}

\author{
C.-K. Sun, J. C. LIANG \\ Department of Electrical Engineering, National Taiwan University, Taipei 10617, Taiwan, Republic of \\ China \\ S. P. DENBAARS \\ Department of Electrical and Computer Engineering, UCSB, Santa Barbara, CA 93106, U.S.A. \\ D. S. KIM, Y. D. CHO \\ Department of Physics, Seoul National University, Seoul 151-742, South Korea \\ G. D. SAnders, J. Simmons, C. J. Stanton \\ Department of Physics, University of Florida, Gainesville, FL 32611, U.S.A.
}

(Received 28 February 2000)

\begin{abstract}
Experiments have shown that ultrafast optical excitation of semiconductors can produce oscillating changes in the optical properties of the material. The frequency of the oscillations in transmission or reflection usually matches one of the phonon modes, typically the $\mathbf{q}=0$ optical mode. These oscillations are known as coherent phonons. We discuss the role of surfaces and interfaces on the coherent phonon signal. We show that: (1) the coherent phonon signal can be used as a probe of the surface depletion field and (2) multiple interfaces as in a superlattice, can drastically alter the coherent phonon spectrum: screening of the modes in the superlattices is reduced and acoustic modes can now be excited.
\end{abstract}

Key words: coherent-phonons, superlattices, ultrafast excitations.

(c) 2000 Academic Press

\section{Introduction}

Femtosecond lasers have proven to be powerful tools for studying the dynamical behavior of photoexcited electrons in semiconductors. Recent experiments have shown that in addition, ultrafast excitation can produce oscillations in the optical transmission or reflection with a frequency matching one of the phonon modes, usually the $\mathbf{q}=0$ optical mode. These oscillations are known as coherent phonons [1-4].

In this paper, we discuss coherent phonons and the effect of surfaces and interfaces on the spectrum of oscillations.

The microscopic theory for non-polar materials is based on a two-band (electron and hole) model with the Hamiltonian given by $[5,6]$

$$
\hat{H}_{e l}=\sum_{\mathbf{k}, \alpha} \epsilon_{\alpha \mathbf{k}} c_{\alpha \mathbf{k}}^{\dagger} c_{\alpha \mathbf{k}}+\sum_{\mathbf{q}} \hbar_{\mathbf{q}} \omega_{\mathbf{q}} b_{\mathbf{q}}^{\dagger} b_{\mathbf{q}}+\sum_{\mathbf{k}, \mathbf{q}, \alpha} M_{\mathbf{k q}}\left(b_{\mathbf{q}}+b_{-\mathbf{q}}^{\dagger}\right) c_{\alpha \mathbf{k}}^{\dagger} c_{\alpha \mathbf{k}+\mathbf{q}}
$$


The first term in eqn (1) represents the Bloch electrons/holes in the noninteracting crystal. Here $c, c^{\dagger}$ are the electron second quantization operators in $\mathbf{k}$-space, $\epsilon_{\alpha \mathbf{k}}$ is the energy dispersion in the conduction and valence bands ( $\alpha=\{c, v\}$ indices the conduction or valence band). The second term represents the optical phonons. Here $\omega_{\mathbf{q}}, b_{\mathbf{q}}^{\dagger}, b_{\mathbf{q}}$ are the phonon dispersion and the phonon creation and annihilation operators. The electrons interact with the optical phonons through the third term. $M_{\mathbf{k q}}$ is the electron-phonon matrix element which typically might describe deformational coupling.

The coherent amplitude of the qth phonon mode is given by:

$$
D_{\mathbf{q}} \equiv\left\langle b_{\mathbf{q}}\right\rangle+\left\langle b_{-\mathbf{q}}^{\dagger}\right\rangle \equiv B_{\mathbf{q}}+B_{-\mathbf{q}}^{*}
$$

and is proportional to Fourier components of the displacement.

One can obtain the equation of motion for the coherent amplitude by commuting eqn (2) with the Hamiltonian eqn (1):

$$
\frac{\partial^{2}}{\partial t^{2}} D_{\mathbf{q}}+\omega_{\mathbf{q}}^{2} D_{\mathbf{q}}=-2 \omega_{\mathbf{q}} \sum_{\alpha, \mathbf{k}} M_{\mathbf{k q}}^{\alpha} n_{\mathbf{k}, \mathbf{k}+\mathbf{q}}^{\alpha} .
$$

This is the equation of a forced oscillator. The right-hand side of eqn (3) depends on the Fourier transform of the electron density matrix and acts as a driving term. When electrons and holes are rapidly created during the laser pulse, this sets off the coherent oscillations. Note that since the laser light is macroscopic, usually the phonon mode that is coherently driven is the $\mathbf{q} \approx 0$ optical mode.

\section{Polar materials}

In polar materials such as GaAs, the coherent phonons are quite pronounced. The mechanism for generation is easily understood. This is illustrated in Fig. 1. A depletion field either from surface states or an externally applied field exists near the surface, as seen in Fig. 1A. This causes the polar lattice to distort in response to the field as shown in Fig. 1C. When electrons and holes are photoexcited by the ultrafast laser pulse, they screen out the depletion field and cause the lattice to relax back to its equilibrium position, Fig. 1B. This triggers oscillations not only in the lattice, but also the electron gas. Details of the interplay between the two are discussed in the paper of Kuznetsov [7, 8].

Two important effects emerge. (i) The amplitude of the coherent phonons depends on the strength of the surface depletion field. (ii) At high carrier excitation density, the photoexcited electrons screen the lattice mode and the frequency shifts from that of the LO mode to that of the TO mode.

\section{Superlattices}

In heterostructures and superlattices, the nature of the coherent phonons can change owing to the multiple interfaces [9]. One major effect that can occur is that the nature of the screening of the lattice modes by the photoexcited carriers can change dramatically. This results from the fact that in a bulk system, the plasmon is optical-like having a finite energy for $\mathbf{q}=0$, while in a two-dimensional (2D) system, the plasmon energy goes to 0 as the wavevector goes to 0 . The plasmon in a $2 \mathrm{D}$ system is unable to screen the LO mode independent of the excitation density. This is illustrated in Fig. 2. In Fig. 2A we show the coherent phonon oscillations in the time domain for bulk $\mathrm{GaAs}$ and a $\mathrm{GaAs} / \mathrm{Al}_{0.36} \mathrm{Ga}_{0.64} \mathrm{As} \mathrm{MQW}$. The beating occurs between the LO and TO modes, originating from the fact that the density profile of the photoexcited carriers is not uniform. In Fig. 2B the Fourier transform in the frequency domain is plotted. One can see that at high carrier densities, the photoexcited electrons can not screen the LO mode as they can in bulk.

Further studies show that the screening of the LO mode in the superlattice depends in detail on the width and height of the barriers. A systematic study has been performed by Yee et al. [10]. These results show that 
(A)

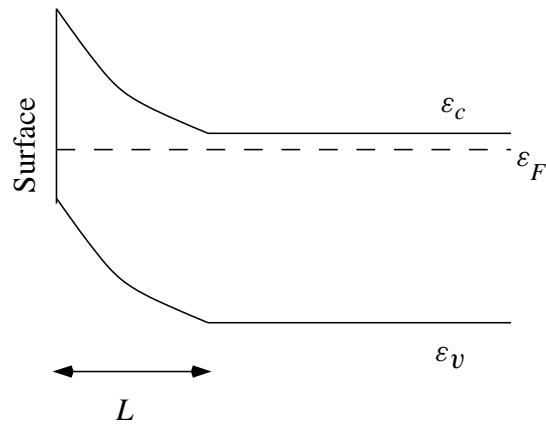

(B)

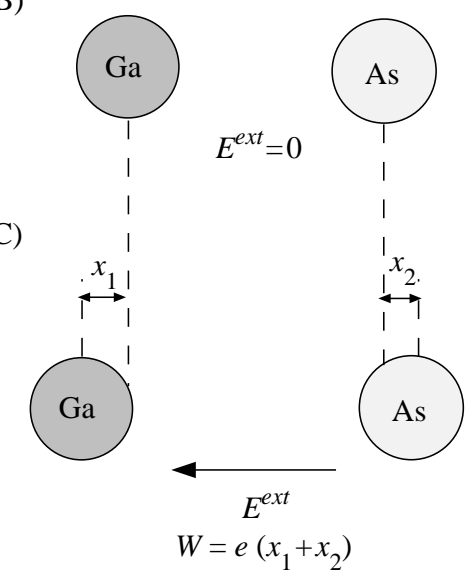

Fig. 1. The energy band diagram of a surface depletion region in GaAs is shown in A. In the depletion region, there are few carriers in a region of length $L$ and large built in fields, $E^{\text {ext }}$. In the large electric field, the Ga and As atoms will move C, with respect to their positions with no electric field $\mathrm{B}$, and create a lattice polarization $W$.

(A)

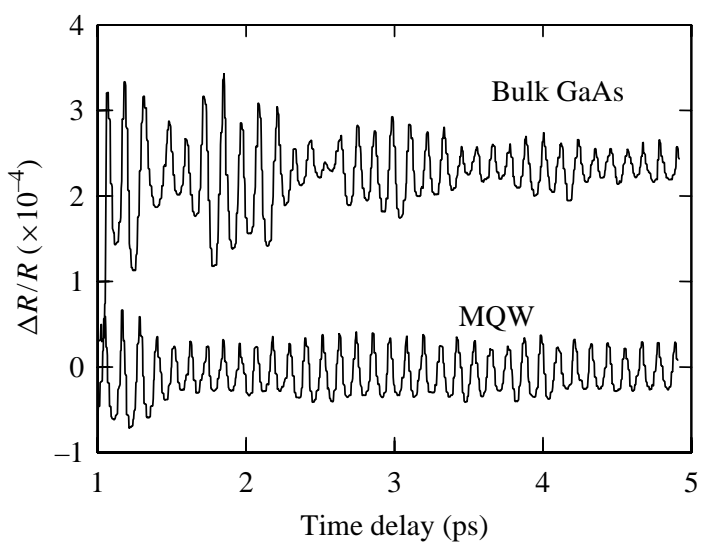

(B)

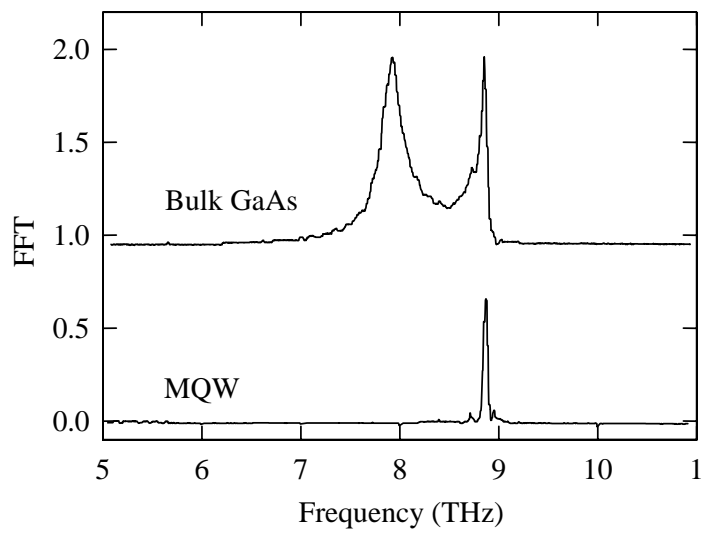

Fig. 2. A, Coherent optical phonon oscillations in the time domain for bulk GaAs and $\mathrm{GaAs}_{\mathrm{Al}} \mathrm{Al}_{0.36} \mathrm{Ga}_{0.64} \mathrm{As} \mathrm{MQW}$ with $L w=15 \mathrm{~nm}$ and $L_{b}=5 \mathrm{~nm}$. B, The Fourier transform in the frequency domain. At high densities, the photoexcited electrons can not screen the LO mode in the MQW since the plasma dispersion varies from the bulk dispersion.

the density dependence of the frequency of oscillation can provide valuable information on the nature and quality of the interfaces in a superlattice.

More interesting behavior occurs in strained layer superlattices such as the InGaN/GaN systems. Here, owing to the wurtzite structure, strain in the superlattice leads to large piezoelectric fields. The large fields lead to strong oscillations, as shown in Fig. 3. These oscillations are so large that they can be seen in transmission and are much stronger than those seen in Fig. 2 in reflection.

Even more interesting is the fact that the oscillations are much slower than in bulk GaAs as can be seen by comparing Figs 2 and 3. In fact, the oscillations are now at the acoustic frequency. The mechanism for the generation of the acoustic phonons can be seen from eqn (3). Since absorption in the superlattice occurs only within the wells, the laser pulse can produce an electron density that has a Fourier component determined by 


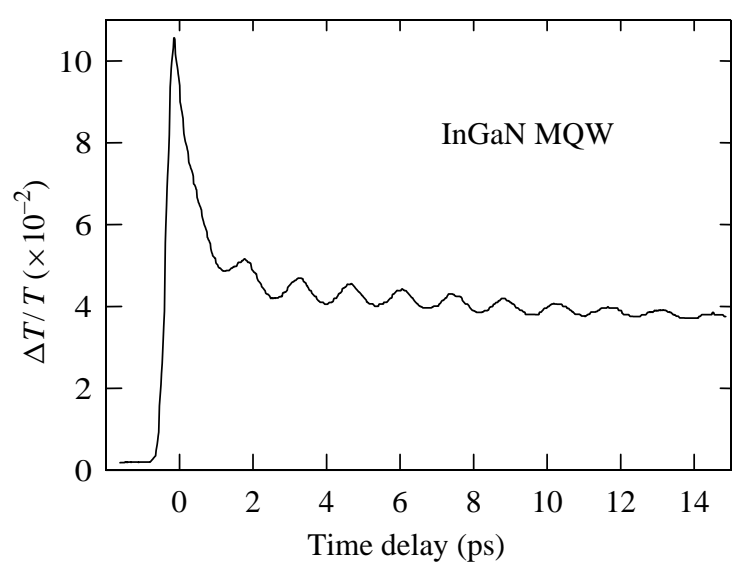

Fig. 3. Transient transmission changes versus probe delay for a 50/43 $\AA$ InGaN/GaN MQW and a laser wavelength of $365 \mathrm{~nm}$. Huge coherent acoustic phonon oscillations are now observed.

the superlattice period. This has been investigated in more detail by Sun et al. [11]. This result shows that the superlattice can significantly change the coherent phonon spectrum and suggests that band-engineering heterostuctures can lead to tailored coherent oscillations.

\section{Conclusions}

We have shown that surfaces and interfaces can alter the properties of the coherent phonons created under ultrafast laser excitation. First, the strength of the oscillations is sensitive to the magnitude of the surface depletion field. Second, interfaces can drastically modify the spectrum: screening of the modes in superlattices for high-density excitation is reduced and acoustic modes can now be excited. This suggests that coherent phonons might be used as sensitive probes of surfaces and interfaces.

Acknowledgements - The authors are grateful to the Aachen group of H. Kurz, especially T. Dekorsey, and to A. Kuznetsov for useful discussions. This work was supported by NSF through grant DMR 9817828 and DOE through grant DE-FG05-91-ER45462.

\section{References}

[1] G. C. Cho, W. Kutt, and H. Kurz, Phys. Rev. Lett. 65, 764 (1990).

[2] W. Kutt, G. C. Cho, T. Pfeifer, and H. Kurz, Semicond. Sci. Technol. B77, 7 (1992).

[3] W. Albrecht, Th. Kruse, and H. Kurz, Phys. Rev. Lett. 69, 1451 (1992).

[4] W. A. Kutt, W. Albrecht, and H. Kurz, IEEE J. Quantum Electronics QE-28, 2434 (1992).

[5] A. V. Kuznetsov and C. J. Stanton, Phys. Rev. Lett. 73, 3243 (1994).

[6] R. Merlin, Solid State Commun. 102, 207 (1997).

[7] A. V. Kuznetsov and C. J. Stanton, Phys. Rev. B52, 7555 (1995).

[8] A. V. Kuznetsov and C. J. Stanton, SPIE 3277, 28 (1998).

[9] T. Dekorsey, A. M. T. Kim, G. C. Cho, H. Kurz, A. V. Kuznetsov, and A. Forster, Phys. Rev. B53, 1531 (1996).

[10] K. J. Yee, D. S. Kim, T. Dekorsey, and Y. S. Lim, Phys. Rev. B60, R8513 (1999).

[11] C.-K. Sun, J.-C. Liang, C. J. Stanton, A. Abare, L. Coldren, and S. DenBaars, Appl. Phys. Lett. 1249, 75 (1999). 